\title{
Balanced Truncation for a Special Class of Bilinear Descriptor Systems
}

\author{
Igor Pontes Duff, Pawan Goyal, and Peter Benner
}

\begin{abstract}
In this paper, we investigate balanced truncation for a class of continuous-time bilinear descriptor systems, appearing, e.g., in constraint circuit analysis or resulting from semi-discretization of the Navier-Stokes equations. For this, as widely done in the literature, we first aim at transforming the bilinear descriptor system into an equivalent ODE system by means of projectors for which the standard balanced truncation method for bilinear systems can be applied. Subsequently, we discuss how to solve the arising generalized Lyapunov equations corresponding to the equivalent $O D E$, without requiring the explicit computation of the projectors. The efficiency of the proposed algorithm is illustrated through a numerical example, which also shows its competitiveness with an iterative $\mathcal{H}_{2}$-optimal model order reduction scheme.
\end{abstract}

Index Terms-Model/controller reduction, large-scale systems, differential-algebraic systems

\section{INTRODUCTION}

$\mathbf{T}$ HIS paper discusses model order reduction based on balanced truncation for bilinear descriptor systems or differential-algebraic equations (DAEs) of the form:

$$
\begin{aligned}
E_{11} \dot{x}_{1}(t)= & A_{11} x_{1}(t)+A_{12} x_{2}(t)+ \\
& \sum_{k=1}^{m} N_{k} x_{1}(t) u_{k}(t)+B_{1} u(t), \\
0= & A_{21} x_{1}(t)+B_{2} u(t), \\
y(t)= & C_{1} x_{1}(t)+C_{2} x_{2}(t),
\end{aligned}
$$

where $x_{1}(t) \in \mathbb{R}^{n_{1}}, x_{2}(t) \in \mathbb{R}^{n_{2}}$ are the state variables, with $n_{1}>n_{2}, u(t) \in \mathbb{R}^{m}$ are the inputs, $y(t) \in \mathbb{R}^{p}$ are the outputs, and $E_{11}, A_{11} \in \mathbb{R}^{n_{1} \times n_{1}}, A_{12}^{T}, A_{21} \in \mathbb{R}^{n_{2} \times n_{1}}, B_{1} \in \mathbb{R}^{n_{1} \times m}$, $B_{2} \in \mathbb{R}^{n_{2} \times m}, C_{1} \in \mathbb{R}^{p \times n_{1}}$ and $C_{2} \in \mathbb{R}^{p \times n_{2}}$. Furthermore, we assume that $E_{11}$ and $A_{21} E_{11}^{-1} A_{12}$ are nonsingular matrices. As a consequence, the linear part of the system (1), i.e., for $N_{k}=0$ with $k=1, \ldots, m$, is a Hessenberg index-2 DAE system, see, e.g., [1]. Generally, these special bilinear DAE systems (1) arise, e.g., from semi-discretization of the NavierStokes equations or constraint RLC circuits.

Model order reduction (MOR) aims at replacing a largescale system by a simpler surrogate low-order system. For an overview of some methods, we refer to the books [2] and [3]. Among the classical techniques, balanced truncation (BT) is one of the popular methods, since it can be easily implemented by solving Lyapunov equations and preserves

Igor Pontes Duff, Pawan Goyal and Peter Benner are with the Computational Methods in Systems and Control Theory (CSC) Group, Max Planck Institute for Dynamics of Complex Technical Systems, Magdeburg, Germany, (emails:\{pontes, goyalp, benner\} (ampi-magdeburg.mpg.de).

Peter Benner is also with the Faculty of Mathematics, Otto von Guericke University, Universitätsplatz 2, 39106 Magdeburg, Germany. asymptotic stability. It was first proposed in [4] in the context of linear time-invariant (LTI) systems, and was later extended to linear DAE systems, see [5]-[7]. Additionally, the author in [8] has considered an extension of BT for a particular class of linear DAE systems, i.e., systems (1) with $N_{k}=0$ for $k=1, \ldots, m$, by using explicit projectors, which can be expensive to compute in a large-scale setting. As a result, the authors in [9] have proposed how to apply BT to the latter systems, avoiding explicit computation of projectors or the related deflating subspaces, and without losing sparsity. Our main goal in this paper is to extend the BT method for bilinear systems [10] to the class of bilinear DAE systems (1). It is worth mentioning that interpolation-based methods for classes of bilinear DAEs including the system (1) can be found in the literature, see [11], [12] and [13].

The rest of the paper is organized as follows. In Section II, we revisit BT for bilinear systems and the associated generalized Lyapunov equations. In Section III, we show how to transform bilinear descriptor systems (1) into an equivalent ordinary differential equation (ODE) form. In Section IV, the main contribution of the paper is established, i.e., the extension of BT for the class of bilinear DAEs (1). Therein, we first develop the method for the equivalent ODE system, and then, we show how to apply the BT method avoiding projection. Finally, in Section V, we carry out some numerical experiments for a medium-scale circuit system, and Section VI concludes the paper.

\section{BALANCED TRUNCATION FOR BILINEAR SYSTEMS}

BT mainly relies on reachability and observability energy functionals. For LTI systems, these functionals are defined via the reachability and observability Gramians. In the context of bilinear systems, algebraic Gramians were first discussed in [14], and later on, in [15], the concepts of reachability and observability are generalized based on the kernels of the corresponding Volterra series. Furthermore, connections of the algebraic Gramians to energy functionals were also studied, see, e.g., [10], [16]. As a result, these Gramians allow us to find the states that are hard to reach as well as hard to observe, which are then truncated to reduce the order of the model.

Moreover, we would like to mention that the concept of energy functionals and balancing-based MOR for general nonlinear, including bilinear, systems was studied in [17]. Therein, it was shown that energy functionals for nonlinear systems are the solutions of state-dependent nonlinear Hamilton-Jacobi equations and Lyapunov-type nonlinear equations. Thus, they are not only difficult to compute for large-scale systems but also hard to apply in the MOR framework. 
In this section, we first briefly overview BT for bilinear ODE systems based on the algebraic Gramians. We begin by considering bilinear ODE systems of the form

$$
\Sigma:\left\{\begin{aligned}
E \dot{x}(t) & =A x(t)+\sum_{k=1}^{m} N_{k} x(t) u_{k}(t)+B u(t), \\
y(t) & =C x(t), \quad x(0)=0,
\end{aligned}\right.
$$

where $x(t) \in \mathbb{R}^{n}, u(t) \in \mathbb{R}^{m}$ and $y(t) \in \mathbb{R}^{p}$ are the state, the input and output vectors of the system, respectively, and all other matrices are of appropriate sizes. Moreover, the matrix $E$ in (2) is considered to be nonsingular, and, hence, the system (2) has no algebraic constraints. In the past years, MOR for bilinear systems has been studied vastly in the literature, see, e.g., [10], [18]-[20]. BT is a projection-based method, i.e., it aims at determining projection matrices $V, W \in \mathbb{R}^{n \times r}$, leading to a reduced-order system

$$
\Sigma_{r}:\left\{\begin{aligned}
\hat{E} \dot{x}(t) & =\hat{A} \hat{x}(t)+\sum_{k=1}^{m} \hat{N}_{k} \hat{x}(t) u_{k}(t)+\hat{B} u(t), \\
\hat{y}(t) & =\hat{C} \hat{x}(t), \quad \hat{x}(0)=0,
\end{aligned}\right.
$$

with $\hat{E}=W^{T} E V, \hat{A}=W^{T} A V, \hat{N}_{k}=W^{T} N_{k} V, \hat{B}=$ $W^{T} B, \hat{C}=C V$. The matrices $V$ and $W$ are constructed by removing the states which are hard to reach as well hard to observe. To that end, we first recall the definition of algebraic Gramians for bilinear ODE systems.

\section{A. Algebraic Gramians for Bilinear ODEs}

The algebraic Gramians, namely reachability $(P)$ and observability $(Q)$ Gramians, for bilinear systems are defined as follows:

$$
\begin{aligned}
& P=\sum_{j=1}^{\infty} \int_{0}^{\infty} \ldots \int_{0}^{\infty} P_{j}\left(t_{1}, \ldots, t_{j}\right) P_{j}\left(t_{1}, \ldots, t_{j}\right)^{T} d t_{1} \ldots d t_{j} \\
& Q=\sum_{j=1}^{\infty} \int_{0}^{\infty} \ldots \int_{0}^{\infty} Q_{j}\left(t_{1}, \ldots, t_{j}\right) Q_{j}\left(t_{1}, \ldots, t_{j}\right)^{T} d t_{1} \ldots d t_{j},
\end{aligned}
$$

respectively, where

$$
\begin{aligned}
& P_{1}\left(t_{1}\right)=e^{E^{-1} A t_{1}} E^{-1} B, \\
& Q_{1}\left(t_{1}\right)=e^{A^{T} E^{-T} t_{1}} C^{T}, \\
& P_{j}\left(t_{1}, \ldots, t_{j}\right)=e^{E^{-1} A t_{j}} E^{-1}\left[N_{1} P_{j-1}, \ldots, N_{m} P_{j-1}\right], \\
& Q_{j}\left(t_{1}, \ldots, t_{j}\right)=e^{A^{T} E^{-T}} t_{j} E^{-T}\left[N_{1}^{T} Q_{j-1}, \ldots, N_{m}^{T} Q_{j-1}\right],
\end{aligned}
$$

for $j \geq 2$. As it can be noticed, the algebraic Gramians (4) are defined as series limits, and thus, they may not exist for a general bilinear system. Hence, sufficient conditions for the existence of these Gramians are studied in [10], [18]. Assuming the Gramians P and Q exist, they solve the following generalized Lyapunov equations

$$
\begin{aligned}
& A P E^{T}+E P A^{T}+\sum_{k=1}^{m} N_{k} P N_{k}^{T}+B B^{T}=0, \\
& A^{T} Q E+E^{T} Q A+\sum_{k=1}^{m} N_{k}^{T} Q N_{k}+C C^{T}=0 .
\end{aligned}
$$

The above equations are also known as Lyapunov-pluspositive, and they also appear in the context of MOR for stochastic systems, see, e.g., [10]. Moreover, the algebraic Gramians (4) provide a (local) lower and upper bound for the reachability and the observability energy functionals, see [10] and [16]. Additionally, the notion of truncated Gramians was studied in [21]. Therein, the authors consider BT using only the first two kernels, i.e., $P_{1}\left(t_{1}\right), P_{2}\left(t_{1}, t_{2}\right), Q_{1}\left(t_{1}\right)$ and $Q_{2}\left(t_{1}, t_{2}\right)$, and perform a study based on it. In what follows, we briefly present some results from the literature for solvers of these generalized Lyapunov equations.

\section{B. Generalized Lyapunov solvers}

Efficient methods for generalized Lyapunov equations (5) are available in the literature, see [22]-[24]. One possible approach to solve them is to use a stationary iterative method, see [22], [24]. The idea behind the technique is to write the generalized Lyapunov equation, e.g., (5a), in the following form:

$$
\mathcal{M}(X)-\mathcal{N}(X)+B B^{T}=0, \text { where }
$$

$\mathcal{M}(X)=A X E^{T}+E X A^{T}$ and $\mathcal{N}(X)=-\sum_{k=1}^{m} N_{k} X N_{k}^{T}$,

and consider a stationary iteration of the form

$$
\mathcal{M}\left(X_{i}\right)=\mathcal{N}\left(X_{i-1}\right)-B B^{T}, \text { for } i=1,2, \ldots
$$

As a consequence, upon convergence, one obtains the solution of the generalized Lyapunov equation. This procedure is a convergent fixed-point iteration if $\rho\left(\mathcal{M}^{-1} \mathcal{N}\right)<1$, see [22]. A common criterion to check convergence is to see if the residual is smaller than a given tolerance, i.e.,

$$
\left\|\operatorname{Res}\left(X_{i}\right)\right\|_{F}=\left\|\mathcal{M}\left(X_{i}\right)-\mathcal{N}\left(X_{i}\right)+B B^{T}\right\|_{F} \leq \text { tol, }
$$

and, once this condition is achieved, the procedure stops. Additionally, in the large-scale setting, the solution is computed as a low-rank approximation, i.e., $X \approx S S^{T}$, with $S \in \mathbb{R}^{n \times k}$ and $k \ll n$.

\section{Balanced truncation for model order reduction}

As mentioned before, the main idea of BT lies in furthermore neglecting the almost unreachable as well as unobservable states, in other words, hard to reach and hard to observe states. In order to guarantee that hard to reach and hard to observe states are truncated simultaneously, we need to find a state transformation $T_{\mathcal{B}}$, such that the bilinear ODE system is transformed into a balanced bilinear system realization. Thus, the reachability and observability Gramians of the transformed realization are the same and diagonal, i.e.,

$$
P=Q=\Sigma=\operatorname{diag}\left(\sigma_{1}, \sigma_{2}, \ldots, \sigma_{n}\right),
$$

where $\sigma_{1} \geq \sigma_{2} \geq \cdots \geq \sigma_{n}>0$ and $\sigma_{k}$ are referred to as the Hankel singular values of the system. Such a transformation exists whenever $P$ and $Q$ are positive definite matrices. Moreover, the small Hankel singular values $\sigma_{k}$ characterize the states that are hard to reach and hard to observe, which can then be truncated. Additionally, the decay of the singular values indicates a good order for a reduced 
Algorithm 1: Balanced truncation for bilinear systems

Input: Matrices $\left(E, A, N_{k}, B, C\right)$ and reduced-order $r$.

Output: Reduced-order matrices $\left(\hat{E}, \hat{A}, \hat{N}_{k}, \hat{B}, \hat{C}\right)$.

1: Compute the low-rank approximation of the Gramians $P \approx S S^{T}$ and $Q \approx R R^{T}$.

2: Compute the SVD of $S^{T} E R$ written as

$$
S^{T} E R=\left[\begin{array}{ll}
U_{1} & U_{2}
\end{array}\right] \operatorname{diag}\left(\Sigma_{1}, \Sigma_{2}\right)\left[\begin{array}{ll}
V_{1} & V_{2}
\end{array}\right]^{T},
$$

with $\Sigma_{1} \in \mathbb{R}^{r \times r}$.

3: Construct the projection matrices $V=S U_{1} \Sigma_{1}^{-\frac{1}{2}}$ and $W=$ $R V_{1} \Sigma_{1}^{\frac{1}{2}}$.

4: Construct $\hat{E}=W^{T} E V, \hat{A}=W^{T} A V, \hat{N}_{k}=W^{T} N_{k} V$, $\hat{B}=W^{T} B$ and $\hat{C}=C V$.

5: return $\hat{E}, \hat{A}, \hat{N}_{k}, \hat{B}$, and $\hat{C}$.

system. A reduced-order system can be computed using the square root balancing approach, which avoids the computation of the balanced realization of the original system (2). The approach is sketched in Algorithm 1. BT for bilinear ODE systems preserves the local stability of the original system, i.e., the matrix $\hat{A}$ is Hurwitz, provided that $A$ is Hurwitz. The method also preserves a stronger notion of stability, see [25] for more details. Additionally, error bounds for the quality of the obtained reduced-order systems can be found in [26]-[28]. In the next section, we discuss how the theory presented in this section can be applied to bilinear DAE systems (1).

\section{TRANSFORMATION OF THE BILINEAR DAE SYSTEM}

In this section, we first discuss a transformation, enabling us to rewrite the bilinear DAE system (1) into an equivalent ODE form. Such a transformation was first introduced in [9], in the context of BT for LTI systems, and later on, it was applied to interpolation-based MOR for bilinear and quadratic-bilinear DAE systems in [13], [29].

We begin with the case $B_{2}=0$ in the system (1); the case $B_{2} \neq 0$ is discussed later in this paper. Thus, we have a system in the following form:

$$
\begin{aligned}
E_{11} \dot{x}_{1}(t)= & A_{11} x_{1}(t)+A_{12} x_{2}(t)+ \\
& \sum_{k=1}^{m} N_{k} x_{1}(t) u_{k}(t)+B_{1} u(t), \\
0= & A_{21} x_{1}(t), \\
y(t)= & C_{1} x_{1}(t)+C_{2} x_{2}(t) .
\end{aligned}
$$

Following the same steps as in [13], from (6b), we have $A_{21} \dot{x}_{1}(t)=0$. Multiplying (6a) by $A_{21} E_{11}^{-1}$ from the left and using the latter relation, we obtain

$$
\begin{aligned}
0=A_{21} & E_{11}^{-1} A_{11} x_{1}(t)+A_{21} E_{11}^{-1} A_{12} x_{2}(t) \\
& +\sum_{k=1}^{m} A_{21} E_{11}^{-1} N_{k} x_{1}(t) u_{k}(t)+A_{21} E_{11}^{-1} B_{1} u(t) .
\end{aligned}
$$

This implies that $x_{2}(t)$ can be written as a function of $x_{1}(t)$. Hence, by inserting this expression into (6a) and (6c), one obtains

$$
\begin{aligned}
E_{11} \dot{x}_{1}(t) & =\Pi A_{11} x_{1}(t)+\sum_{k=1}^{m} \Pi N_{k} x_{1}(t) u_{k}(t)+\Pi B_{1} u(t), \\
y(t) & =\mathcal{C} x_{1}(t)+\sum_{k=1}^{m} \mathcal{C}_{N}^{(k)} x_{1}(t) u_{k}(t)
\end{aligned}
$$

where

$$
\Pi=I-A_{12}\left(A_{21} E_{11}^{-1} A_{12}\right)^{-1} A_{21} E_{11}^{-1},
$$

and

$$
\begin{aligned}
\mathcal{C} & =C_{1}-C_{2}\left(A_{12} E_{11}^{-1} A_{12}\right) A_{12} E_{11}^{-1} A_{11}, \\
\mathcal{C}_{N}^{(k)} & =-C_{2}\left(A_{12} E_{11}^{-1} A_{12}\right)^{-1} A_{12} E_{11}^{-1} N_{k} .
\end{aligned}
$$

For simplicity, we assume that $A_{12}=A_{21}^{T}$. However, $A_{21} \neq$ $A_{12}^{T}$ can also be handled similarly by extending the arguments used in [30]. As shown in [9], $\Pi$ is an oblique projector, satisfying

$$
A_{12}^{T} z=0 \text { if and only if } \Pi^{T} z=z .
$$

As a consequence of $(6 \mathrm{~b}), \Pi^{T} x_{1}(t)=x_{1}(t)$. By replacing $x_{1}(t)$ by $\Pi^{T} x_{1}(t)$ and multiplying (7) by $\Pi$ from the left, we obtain

$$
\begin{aligned}
E_{\Pi} \dot{x}_{1}(t) & =A_{\Pi} x_{1}(t)+\sum_{k=1}^{m} N_{\Pi, k} x_{1}(t) u_{k}(t)+B_{\Pi} u(t), \\
y(t) & =C_{\Pi} x_{1}(t)+\sum_{k=1}^{m} \mathcal{C}_{N, \Pi}^{(k)} x_{1}(t) u_{k}(t),
\end{aligned}
$$

where $E_{\Pi}=\Pi E_{11} \Pi^{T}, A_{\Pi}=\Pi A_{11} \Pi^{T}, N_{\Pi, k}=\Pi N_{k} \Pi^{T}$, $B_{\Pi}=\Pi B_{1}, C_{\Pi}=C \Pi^{T}$ and $\mathcal{C}_{N, \Pi}^{(k)}=\mathcal{C}_{N}^{(k)} \Pi^{T}$. As stated in [13], the bilinear system (9) evolves in the $n_{1}-n_{2}$ dimensional subspace, belonging to $\operatorname{ker}(\Pi)$. This can be made more explicit by expressing

$$
\Pi=\Theta_{l} \Theta_{r}^{T},
$$

with $\Theta_{l}, \Theta_{r} \in \mathbb{R}^{n_{1} \times\left(n_{1}-n_{2}\right)}$, such that $\Theta_{l}^{T} \Theta_{r}=I$. Substituting this decomposition in (9), $\tilde{x}_{1}:=\Theta_{l}^{T} x_{1} \in \mathbb{R}^{n_{1}-n_{2}}$ satisfies the following equation:

$$
\begin{aligned}
E_{\Theta} \dot{\tilde{x}}_{1} & =A_{\Theta} \tilde{x}_{1}+\sum_{k=1}^{m} N_{\Theta, k} \tilde{x}_{1}(t) u_{k}(t)+B_{\Theta} u(t), \\
y(t) & =\mathcal{C}_{\Theta} \tilde{x}_{1}(t)+\sum_{k=1}^{m} \mathcal{C}_{N, \Theta}^{(k)} \tilde{x}_{1}(t) u_{k}(t),
\end{aligned}
$$

where $E_{\Theta}=\Theta_{r}^{T} E_{11} \Theta_{r}, A_{\Theta}=\Theta_{r}^{T} A_{11} \Theta_{r}, N_{\Theta, k}=\Theta_{r}^{T} N_{k} \Theta_{r}$ $B_{\Theta}=\Theta_{r}^{T} B_{1}, C_{\Theta}=C \Theta_{r}$ and $\mathcal{C}_{N, \Theta}^{(k)}=\mathcal{C}_{N}^{(k)} \Theta_{r}$.

The system (11) is equivalent to the system (6) with respect to the input and output behavior. However, an advantage of the realization given by (11) is that the matrix $E_{\Theta}$ is nonsingular, since $\Theta_{r}$ has full column rank and $E_{11}$ is nonsingular. But the output given by (11b) involves bilinear terms in the state and input. Since treating bilinear terms in the output equation is still an open problem, we will not consider the terms $\mathcal{C}_{N, \Theta}^{(k)}$ while computing the projection matrices $V$ and $W$. Hence, from now on, we assume $\mathcal{C}_{N, \Theta}^{(k)}=0$ as far as 
$\overline{\text { Algorithm 2: BT for bilinear descriptor systems involving }}$ projectors

Input: $E_{11}, A_{11}, N_{k}, B_{1}, C, \mathcal{C}_{N}^{(k)}$ and order $r$.

1: Compute $\Theta_{r}, \Theta_{l}$, and the matrices $E_{\Theta}, A_{\Theta}, N_{\Theta, k}, B_{\Theta}$, $C_{\Theta}$ and $\mathcal{C}_{N, \Theta}^{(k)}$.

2: Compute low-rank factors of $P \approx \tilde{S} \tilde{S}^{T}$ and $Q \approx \tilde{R} \tilde{R}^{T}$ as solution of the Lyapunov equations (12).

3: Compute the SVD of $\tilde{S}^{T} E_{\Theta} \tilde{R}$ written as

$$
\tilde{S}^{T} E_{\Theta} \tilde{R}=\left[\begin{array}{ll}
U_{1} & U_{2}
\end{array}\right] \operatorname{diag}\left(\Sigma_{1}, \Sigma_{2}\right)\left[\begin{array}{ll}
V_{1} & V_{2}
\end{array}\right]^{T},
$$

with $\Sigma_{1} \in \mathbb{R}^{r \times r}$.

4: Construct the projection matrices $\tilde{V}=\tilde{S} U_{1} \Sigma_{1}^{-\frac{1}{2}}$ and $W=$ $\tilde{R} V_{1} \Sigma_{1}^{\frac{1}{2}}$.

Output: reduced order matrices

$\hat{E}=\tilde{W}^{T} E_{\Theta} \tilde{V}, \hat{A}=\tilde{W}^{T} A_{\Theta} \tilde{V}, \hat{N}_{k}=\tilde{W}^{T} N_{\Theta, k} \tilde{V}, \hat{B}=$ $\tilde{W}^{T} B_{\Theta}, \hat{C}=C_{\Theta} \tilde{V}$ and $\hat{\mathcal{C}}_{N}^{(k)}=\mathcal{C}_{N, \Theta}^{(k)} \tilde{V}$.

determining projection matrices for MOR; but note that the bilinear terms in the output equation are projected afterward using the obtained projection matrices. Hence, this allows us to obtain a bilinear system whose realization has a similar form as given in (2), enabling us to apply BT as presented in Section II. In the next section, we discuss two different procedures to apply BT to bilinear DAE systems of form (6) or its equivalent representation (11).

\section{BALANCED TRUNCATION FOR BILINEAR DAE SYSTEMS OF INDEX-2}

\section{A. Balanced truncation involving projector}

We recall, under the assumption $\mathcal{C}_{N, \Theta}^{(k)}=0$, that the realization given by (11) is equivalent to the one from (6). Hence, applying BT to the system (11) requires computation of the reachability and observability Gramians $\tilde{P}, \tilde{Q} \in$ $\mathbb{R}^{\left(n_{1}-n_{2}\right) \times\left(n_{1}-n_{2}\right)}$, which are the solutions of the following equations:

$$
\begin{aligned}
& A_{\Theta} \tilde{P} E_{\Theta}^{T}+E_{\Theta} \tilde{P} A_{\Theta}^{T}+\sum_{k=1}^{m} N_{\Theta, k} \tilde{P} N_{\Theta, k}^{T}+B_{\Theta} B_{\Theta}^{T}=0, \\
& A_{\Theta}^{T} \tilde{Q} E_{\Theta}+E_{\Theta}^{T} \tilde{Q} A_{\Theta}+\sum_{k=1}^{m} N_{\Theta, k}^{T} \tilde{Q} N_{\Theta, k}+C_{\Theta}^{T} C_{\Theta}=0 .
\end{aligned}
$$

Thus, by following the BT procedure presented in Subsection II-C, one is able to find a reduced-order system. Algorithm 2 summarizes the steps to construct a reduced-order system for the system (6) based on the Gramians, given in (12). Unfortunately, to determine the system matrices of (11), we require the explicit computation of the matrices $\Theta_{r}$ and $\Theta_{l}$, which might be an expensive computational task. Moreover, transforming the realization (6) into the system (11) might imply the loss of sparsity, thus making the computation of the reduced-order systems more expensive. Therefore, rather than reducing system (11), we aim at reducing (6) or (9), without explicit computation of the matrices $\Pi, \Theta_{r}$, and $\Theta_{l}$. That is precisely the scope of the next subsection.

\section{B. Projector-free balanced truncation}

We aim at constructing a reduced-order system without explicitly computing the matrices $\Theta_{l}$ and $\Theta_{r}$, and the system matrices of (11). By adapting the steps from [9] to the bilinear context, our first goal is to establish generalized Lyapunov equations for $P:=\Theta_{r} \tilde{P} \Theta_{r}^{T}, Q:=\Theta_{r} \tilde{Q} \Theta_{r}^{T}$. Using the relation $\Theta_{l}^{T} \Theta_{r}=I$, it can easily be seen that the matrices $P$ and $Q$ satisfy the following identities:

$$
P=\Pi^{T} P \Pi, \quad Q=\Pi^{T} Q \Pi .
$$

Moreover, having multiplied (12a) by $\Theta_{r}$ from the left and right, and (12b) from the left and right by $\Theta_{l}$, we notice that $P$ and $Q$ solve the following projected Lyapunov equations:

$$
\begin{aligned}
& A_{\Pi} P E_{\Pi}^{T}+E_{\Pi} P A_{\Pi}^{T}+\sum_{k=1}^{m} N_{\Pi, k} P N_{\Pi, k}^{T}+B_{\Pi} B_{\Pi}^{T}=0, \\
& A_{\Pi}^{T} Q E_{\Pi}+E_{\Pi}^{T} Q A_{\Pi}+\sum_{k=1}^{m} N_{\Pi, k}^{T} Q N_{\Pi, k}+C_{\Pi}^{T} C_{\Pi}=0,
\end{aligned}
$$

where $\Pi=I-A_{12}\left(A_{12}^{T} E_{11}^{-1} A_{12}\right)^{-1} A_{12}^{T} E_{11}^{-1}$. In [9], projected ADI Lyapunov solvers for the linearized version of (13) are presented, i.e., the case where $N_{\Pi, k}=0$ for $k=1, \ldots, m$. This enables us to compute low-rank approximations to the solution of the equations

$$
\begin{aligned}
& A_{\Pi} P_{l} E_{\Pi}^{T}+E_{\Pi} P_{l} A_{\Pi}^{T}+B_{\Pi} B_{\Pi}^{T}=0, \\
& A_{\Pi}^{T} Q_{l} E_{\Pi}+E_{\Pi}^{T} Q_{l} A_{\Pi}+C_{\Pi}^{T} C_{\Pi}=0,
\end{aligned}
$$

i.e., $P_{l} \approx S_{l} S_{l}^{T}$ and $Q_{l} \approx R_{l} R_{l}^{T}$, with $S_{l} \in \mathbb{R}^{n \times k_{P}}$, $R_{l} \in \mathbb{R}^{n \times k_{Q}}$, and $k_{P}, k_{Q} \ll n$. This procedure uses only the original system matrices $E_{11}, A_{11}, A_{12}, B$, and $C$, without explicitly computing the projector $\Pi$. As a consequence, the solver takes advantage of the sparsity of those matrices and it is numerically less expensive than dealing directly with the projected system (11). For details, see [9, Sec. 5].

Our approach to solve (13) is to combine the above proposed projected ADI Lyapunov solver with a stationary iterative method for generalized Lyapunov equations (sketched in Subsection II-B). In what follows, we focus on the computation of the reachability Gramian, and the observability Gramian will follow similarly. To that end, let us define the following operators

$$
\begin{aligned}
\mathcal{M}_{\Pi}(X) & =A_{\Pi} X E_{\Pi}^{T}+E_{\Pi} X A_{\Pi}^{T} \text { and } \\
\mathcal{N}_{\Pi}(X) & =-\sum_{k=1}^{m} N_{\Pi, k} X N_{\Pi, k}^{T},
\end{aligned}
$$

and consider a stationary iteration of the form

$$
\mathcal{M}_{\Pi}\left(X_{i}\right)=\mathcal{N}_{\Pi}\left(X_{i-1}\right)-B_{\Pi} B_{\Pi}^{T}, \text { for } i=1,2, \ldots
$$

Let $X_{i-1} \approx S_{i-1} S_{i-1}^{T}$ be the factorized form of the solution obtained in the $(i-1)$ st iteration. Hence,

$$
X_{i-1}=\Pi^{T} X_{i-1} \Pi \approx \Pi^{T} S_{i-1} S_{i-1}^{T} \Pi .
$$

As a consequence, at every iteration step, one needs to solve the following Lyapunov equation:

$$
A_{\Pi} X_{i} E_{\Pi}^{T}+E_{\Pi} X_{i} A_{\Pi}^{T}+\mathcal{B}_{\Pi, i} \mathcal{B}_{\Pi, i}^{T}=0,
$$


Algorithm 3: Iterative ADI based solver for (13)

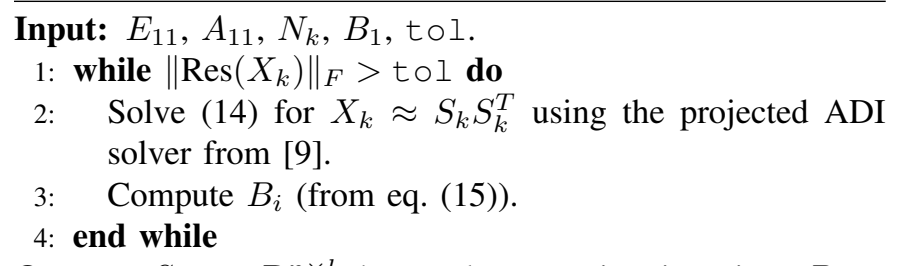

Output: $S_{k} \in \mathbb{R}^{n \times l}$ low-rank approximation, i.e., $P \approx$ $S_{k} S_{k}^{T}$.

Algorithm 4: BT for bilinear DAE systems without projector

Input: $E_{11}, A_{11}, N_{k}, B_{1}, \mathcal{C}, \mathcal{C}_{N}^{(k)}$ and order $r$.

1: Compute low-rank approximations of $P \approx S S^{T}$ and $Q \approx$ $R R^{T}$ from (13) using Algorithm 3.

2: Compute the SVD of $S^{T} E_{11} R$ written as

$$
S^{T} E_{11} R=\left[\begin{array}{ll}
U_{1} & U_{2}
\end{array}\right] \operatorname{diag}\left(\Sigma_{1}, \Sigma_{2}\right)\left[\begin{array}{ll}
V_{1} & V_{2}
\end{array}\right]^{T},
$$

with $\Sigma_{1} \in \mathbb{R}^{r \times r}$.

Output: reduced order matrices:

$\hat{E}=W^{T} E_{11} V, \hat{A}=W^{T} A_{11} V, \hat{N}_{k}=W^{T} N_{k} V, \hat{B}=$ $W^{T} B_{1}, \hat{C}=\mathcal{C} V$ and $\hat{C}_{N}^{(k)}=\mathcal{C}_{N}^{(k)} V$.

$$
\text { where } \mathcal{B}_{\Pi, 1}=\Pi^{T} B_{1} \text {, and } \mathcal{B}_{\Pi, i}=\Pi^{T} B_{i} \text {, }
$$

for $i \geq 2$, with $B_{i}=\left[N_{1} S_{i-1}, N_{2} S_{i-1}, \ldots, N_{m} S_{i-1}, B_{1}\right]$. Hence, by using the projected ADI solver, one can compute the solution of (14) as a low-rank approximation $X_{i} \approx S_{i} S_{i}^{T}$, without the explicit computation of the projector $\Pi$, and the matrices $\Theta_{r}$ and $\Theta_{l}$. We only need at each iteration the matrices $E_{11}, A_{11}, A_{12}$, and $B_{i}$. The procedure keeps iterating until the residual is smaller than a tolerance, i.e.,

$$
\left\|\operatorname{Res}\left(X_{i}\right)\right\|_{F}=\left\|\mathcal{M}_{\Pi}\left(X_{i}\right)-\mathcal{N}_{\Pi}\left(X_{i}\right)+B_{\Pi} B_{\Pi}^{T}\right\|_{F} \leq \text { tol. }
$$

Algorithm 3 summarizes the computation of the low-rank solution of (13), and Algorithm 4 shows the steps to construct a reduced-order system for the system (6) without explicit computation of projectors or its basis.

Remark 1: Both Algorithms 2 and 4 preserve local asymptotic stability of the original systems. This is due to the fact that these algorithms are equivalent to applying BT to the projected ODE system, which, in turn, preserves local asymptotic stability.

Remark 2: The case $B_{2} \neq 0$ has been discussed in [9], for linear systems, and in [13], for bilinear systems. Therein, it has been shown how to equivalently rewrite a system with $B_{2} \neq 0$ as a system with $B_{2}=0$. For more details, we refer to [13] and [9, Sec. 6].

\section{NUMERICAL EXPERIMENT}

In this section, we test the efficiency of the proposed BT method for bilinear DAE systems, having an index-2 matrix pencil. For the projected ADI Lyapunov solvers, we use the implementation provided in the M-M.E.S.S. toolbox [31]. We compare the results with the ones obtained by applying the $\mathcal{H}_{2}$-optimal MOR method proposed in [13] for the same class of systems. All the simulations are done on a

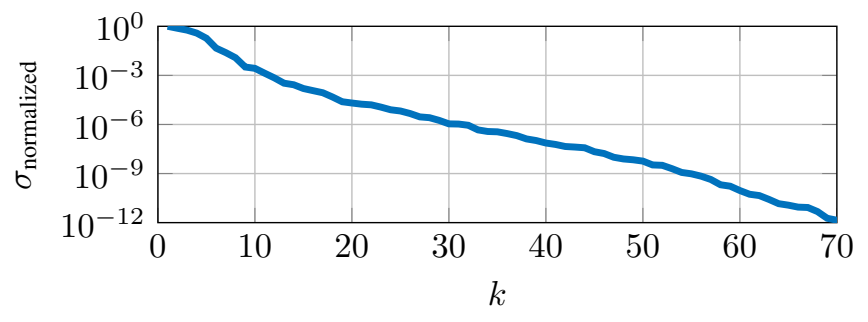

Fig. 1: Decay of normalized Hankel singular values.

CPU 2.6 GHz Intel ${ }^{\circledR}$ Core $^{\mathrm{TM}} \mathrm{i} 5,8 \mathrm{~GB} 1600 \mathrm{MHz}$ DDR3, MATLAB $^{\circledR}$ 9.1.0.441655 (R2016b).

We consider a nonlinear RC circuit presented in [13, Sec. 5]. It consists of a constraint transmission line circuit, where the voltages at the first and last nodes are the same. The nonlinearities come from diodes modeled as $g\left(v_{D}\right)=e^{40 v_{D}}+v_{D}-1$, where $v_{D}$ is the voltage across the node. In this example, $y(t)$ correspond to the voltage at the first node. Having applied a Carleman bilinearization [13], we end up with a bilinear descriptor system of the form (1) with $n_{1}=930$ and $n_{2}=31$. In what follows, we refer to the obtained bilinear descriptor system as the original one.

We now aim at obtaining reduced systems of orders $r=10$ and $r=20$ by using the proposed BT and the $\mathcal{H}_{2}$-optimal MOR methods. Figure 1 depicts the decay of the Hankel singular values. In Figures $2 a$ and $2 b$, we compare the quality of the reduced-order systems with the original system by computing transient responses for an input $u(t)=(\sin (10 \pi t)+1) / 2$. To determine the transient response, we apply an implicit Euler scheme with step size $h=0.01$ and simulate the original system and the reduced-order models (ROMs) in the time interval $[0,10]$. The outputs of the original system and the ROMs are depicted in Figure 2a. Additionally, in Figure 2b, we present the relative error between the original and the ROM, i.e., $\left(\left|y_{\text {Ori }}(t)-y_{\mathrm{ROM}}(t)\right|\right) / \max _{t} \mid y_{\text {Ori }}(t)$. Although all ROMs are able to follow the behavior of the original system, the ROMs of order 20 are of better quality than those of order 10 as expected. Additionally, these figures show that the two methods, BT and $\mathcal{H}_{2}$-optimal MOR methods, provide very similar quality ROMs in terms of the magnitude of the error. However, we note that BT has the advantage of providing an indicator of a good order of a reduced-order system $r$, by observing the decay of the singular values, i.e., one can determine the order of the ROM automatically rather than picking $r$ beforehand.

\section{Conclusions}

In this paper, we have studied the problem of balanced truncation for bilinear descriptor systems of index-2. To that aim, by explicitly enforcing the algebraic conditions, the original descriptor system is first transformed into an equivalent bilinear ODE system by using projectors. This has enabled us to apply the standard BT method for bilinear ODE systems. However, this process is computationally expensive due to the explicit involvement of the projector. To cope with this issue, we have proposed a BT method for the class of bilinear DAEs, avoiding the explicit computation of projectors in order to compute reduced-order systems. Finally, we have illustrated 


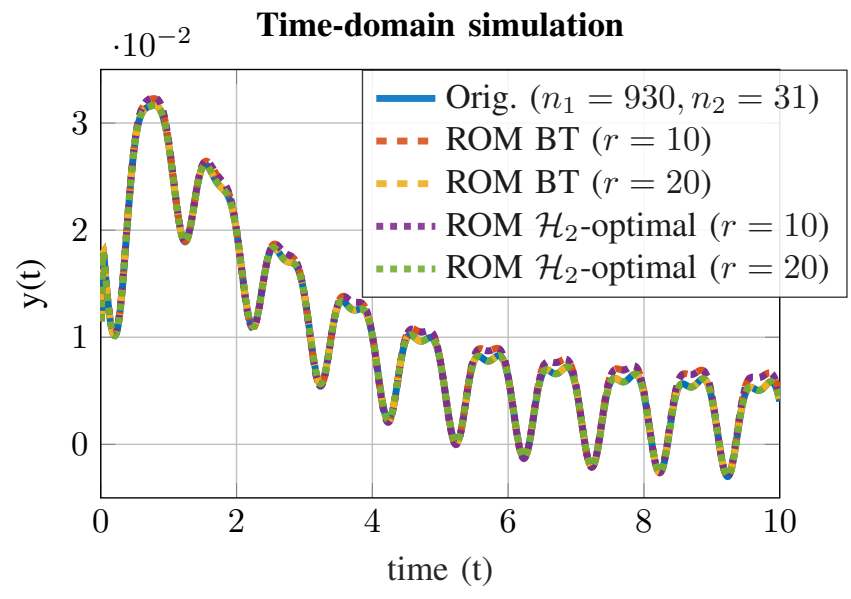

(a) Transient response.

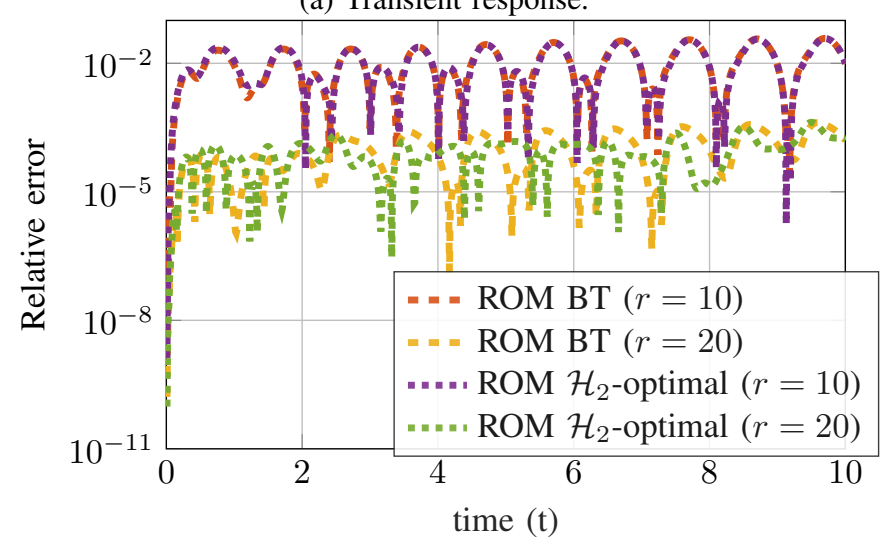

(b) Relative error.

Fig. 2: Comparison of the time-domain simulations of the original and ROMs of orders $r=10$ and $r=20$, obtained using BT and $\mathcal{H}_{2}$-optimal MOR methods.

the efficiency of the proposed approach using a nonlinear electrical circuit example and have shown its competitiveness with an iterative $\mathcal{H}_{2}$-optimal model order reduction scheme.

\section{REFERENCES}

[1] E. Hairer and G. Wanner, Solving Ordinary Differential Equations II-Stiff and Differential-Algebraic Problems, ser. Springer Series in Computational Mathematics 14. Berlin: Springer-Verlag, 1991.

[2] A. C. Antoulas, Approximation of Large-Scale Dynamical Systems, ser. Advances in Design and Control. Philadelphia, PA: SIAM Publications, 2005, vol. 6.

[3] P. Benner, A. Cohen, M. Ohlberger, and K. Willcox, Eds., Model Reduction and Approximation: Theory and Algorithms, ser. Computational Science \& Engineering. Philadelphia, PA: SIAM Publications, 2017.

[4] B. C. Moore, "Principal component analysis in linear systems: controllability, observability, and model reduction," IEEE Trans. Autom. Control, vol. AC-26, no. 1, pp. 17-32, 1981.

[5] W. Q. Liu and V. Sreeram, "Model reduction of singular systems," Internat. J. Sys. Sci., vol. 32, no. 10, pp. 1205-1215, 2001.

[6] T. Stykel, "Analysis and numerical solution of generalized Lyapunov equations," Ph.D. Thesis, Technische Universität Berlin, Berlin, 2002.

[7] V. Mehrmann and T. Stykel, "Balanced truncation model reduction for large-scale systems in descriptor form," in Dimension Reduction of Large-Scale Systems, ser. Lect. Notes Comput. Sci. Eng., P. Benner, V. Mehrmann, and D. C. Sorensen, Eds., vol. 45. Springer-Verlag, Berlin/Heidelberg, Germany, 2005, pp. 83-115.

[8] T. Stykel, "Balanced truncation model reduction for semidiscretized Stokes equation," Linear Algebra Appl., vol. 415, no. 2-3, pp. 262-289, 2006.
[9] M. Heinkenschloss, D. C. Sorensen, and K. Sun, "Balanced truncation model reduction for a class of descriptor systems with application to the Oseen equations," SIAM J. Sci. Comput., vol. 30, no. 2, pp. 1038-1063, 2008.

[10] P. Benner and T. Damm, "Lyapunov equations, energy functionals, and model order reduction of bilinear and stochastic systems," SIAM J. Cont. Optim., vol. 49, no. 2, pp. 686-711, 2011.

[11] M. I. Ahmad, P. Benner, and P. Goyal, "Krylov subspace-based model reduction for a class of bilinear descriptor systems," J. Comput. Appl. Math., vol. 315, pp. 303-318, 2017.

[12] P. Benner and P. Goyal, "Multipoint interpolation of Volterra series and $\mathcal{H}_{2}$-model reduction for a family of bilinear descriptor systems," Syst. Control Lett., vol. 97, pp. 1-11, 2016.

[13] P. Goyal and P. Benner, "An iterative model order reduction scheme for a special class of bilinear descriptor systems appearing in constraint circuit simulation," in ECCOMAS Congress 2016, VII European Congress on Computational Methods in Applied Sciences and Engineering, vol. 2, 2016, pp. 4196-4212. [Online]. Available: https://drive.google.com/open?id=0B04URIVl3wyuVHhMeFVBSldGX2c

[14] P. D'Alessandro, A. Isidori, and A. Ruberti, "Realization and structure theory of bilinear dynamical systems," SIAM J. Cont., vol. 12, no. 3, pp. 517-535, 1974.

[15] S. A. Al-Baiyat and M. Bettayeb, "A new model reduction scheme for k-power bilinear systems," in Proc. of the 32nd IEEE Conference on Decision and Control. IEEE, 1993, pp. 22-27.

[16] P. K. Goyal, "System-theoretic model order reduction for bilinear and quadratic-bilinear systems," Ph.D. Thesis, Otto-von-GuerickeUniversität, Magdeburg, Germany, 2018.

[17] J. M. A. Scherpen, "Balancing for nonlinear systems," Syst. Control Lett., vol. 21, no. 2, pp. 143-153, 1993.

[18] L. Zhang and J. Lam, "On $\mathrm{H}_{2}$ model reduction of bilinear systems," Automatica, vol. 38, no. 2, pp. 205-216, 2002.

[19] Z. Bai and D. Skoogh, "A projection method for model reduction of bilinear dynamical systems," Linear Algebra Appl., vol. 415, no. 2-3, pp. 406-425, 2006.

[20] J. R. Phillips, "Projection-based approaches for model reduction of weakly nonlinear, time-varying systems," IEEE Trans. Comput.-Aided Design Integr. Circuits Syst., vol. 22, no. 2, pp. 171-187, 2003.

[21] P. Benner, P. Goyal, and M. Redmann, "Truncated Gramians for bilinear systems and their advantages in model order reduction," in Model Reduction of Parametrized Systems, ser. MS\&A - Modeling, Simulation and Applications, P. Benner, M. Ohlberger, T. Patera, G. Rozza, and K. Urban, Eds. Cham: Springer, 2017, vol. 17, pp. 285-300.

[22] T. Damm, "Direct methods and ADI-preconditioned Krylov subspace methods for generalized Lyapunov equations," Numer. Lin. Alg. Appl., vol. 15 , no. 9, pp. 853-871, 2008 .

[23] P. Benner and T. Breiten, "Low rank methods for a class of generalized Lyapunov equations and related issues," Numer. Math., vol. 124, no. 3 , pp. 441-470, 2013.

[24] S. D. Shank, V. Simoncini, and D. B. Szyld, "Efficient low-rank solution of generalized Lyapunov equations," Numer. Math., vol. 134, no. 2, pp. 327-342, 2016.

[25] P. Benner, T. Damm, M. Redmann, and Y. R. Rodriguez Cruz, "Positive operators and stable truncation," Linear Algebra Appl., vol. 498, pp. 74-87, 2016.

[26] S. Becker and C. Hartmann, "Infinite-dimensional bilinear and stochastic balanced truncation with error bounds," arXiv e-prints, arXiv:1806.05322, June 2018. [Online]. Available: https://arxiv.org/abs/1806.05322

[27] P. Benner, T. Damm, and Y. R. Rodriguez Cruz, "Dual pairs of generalized Lyapunov inequalities and balanced truncation of stochastic linear systems," IEEE Trans. Autom. Control, vol. 62, no. 2, pp. 782791, 2017.

[28] M. Redmann, "Type II balanced truncation for deterministic bilinear control systems," SIAM J. Cont. Optim., vol. 56, no. 4, pp. 2593-2612, 2018.

[29] M. I. Ahmad, P. Benner, P. Goyal, and J. Heiland, "Moment-matching based model reduction for Navier-Stokes type quadratic-bilinear descriptor systems," Z. Angew. Math. Mech., vol. 97, no. 10, pp. 1252-1267, 2017.

[30] S. Gugercin, T. Stykel, and S. Wyatt, "Model reduction of descriptor systems by interpolatory projection methods," SIAM J. Sci. Comput., vol. 35, no. 5, pp. B1010-B1033, 2013.

[31] J. Saak, M. Köhler, and P. Benner, "M-M.E.S.S.-1.0.1 - The Matrix Equation Sparse Solver Library," Apr. 2016. [Online]. Available: https://doi.org/10.5281/zenodo.50575 